\title{
How We Established a Multidisciplinary Program for Vascular Anomalies
}

\author{
Alexandra Borst ${ }^{1}$, Rachel Swerdlin² ${ }^{2}$ James Phillips ${ }^{3}$, Clifford Hawkins ${ }^{4}$, and Michael \\ Briones $^{5}$ \\ ${ }^{1}$ Monroe Carell Junior Children's Hospital at Vanderbilt \\ ${ }^{2}$ Children's Healthcare of Atlanta Inc \\ ${ }^{3}$ Vanderbilt University Medical Center \\ ${ }^{4}$ Emory Healthcare \\ ${ }^{5}$ Emory University
}

October 28, 2020

\begin{abstract}
The care of patients with vascular anomalies is quickly becoming a complex field requiring high quality, coordinated multidisciplinary care. In this article, we review the history of multidisciplinary care in this field, discuss the benefits of this model of care, and outline some of the essential components and structure of a successful vascular anomalies team. We provide an overview of two example programs and a roadmap for other centers to develop their own multidisciplinary vascular anomalies teams.
\end{abstract}

Title: How We Established a Multidisciplinary Program for Vascular Anomalies

Authors: Alexandra J. Borst, MD ${ }^{1}$, Rachel F. Swerdlin, CPNP ${ }^{2}$, James D. Phillips ${ }^{1}$, MD, C. Matthew Hawkins $^{3}$, MD, and Michael A. Briones, $\mathrm{DO}^{4}$

${ }^{1}$ Divisons of Pediatric Hematology-Oncology and Pediatric Otolaryngology, Monroe Carrell Jr Children's Hospital, Vanderbilt University Medical Center, Nashville, TN

${ }^{2}$ Vascular Anomalies Clinic, Children's Healthcare of Atlanta, Atlanta, GA

${ }^{3}$ Department of Radiology and Imaging Services, Children's Healthcare of Atlanta, Emory University Medical Center and Children's Pediatric Institute, Atlanta, GA

${ }^{4}$ Division of Pediatric Hematology-Oncology, Children's Healthcare of Atlanta, Emory University Medical Center and Children's Pediatric Institute, Atlanta, GA

\section{Corresponding Author:}

Alexandra J. Borst, MD

Division of Pediatric Hematology-Oncology

Vanderbilt University Medical Center

2200 Children's Way, $6^{\text {th }}$ Floor DOT

Nashville, TN 37232

Phone: 615-936-1762 
Fax: $615-936-1767$

Email: alexandra.borst@vumc.org

Word Count Abstract: 77

Word Count Main Text: 3,844

Number of Tables: 3

Number of Figures: 3

Running Title: Multidisciplinary Vascular Anomalies Program

Keywords: vascular anomaly, program, malformation, model, multidisciplinary

\section{Abbreviations:}

\begin{tabular}{ll}
\hline APP & advanced practice provider \\
\hline CHOA & Children's Healthcare of Atlanta \\
HMO & health maintenance organizations \\
ISSVA & International Society of the Study of Vascular Anomalies \\
MDC & multidisciplinary clinic \\
NP & nurse practitioner \\
OT & occupational therapy \\
PA & physician's assistant \\
PT & Physical therapy \\
QOL & quality of life \\
VA-MDC & vascular anomalies multidisciplinary clinic \\
\hline
\end{tabular}

\section{Abstract}

The care of patients with vascular anomalies is quickly becoming a complex field requiring high quality, coordinated multidisciplinary care. In this article, we review the history of multidisciplinary care in this field, discuss the benefits of this model of care, and outline some of the essential components and structure of a successful vascular anomalies team. We provide an overview of two example programs and a roadmap for other centers to develop their own multidisciplinary vascular anomalies teams.

\section{Main Text}

\section{Historical Perspective}

The role of physicians in the care of children with vascular birthmarks and malformations can be traced back for centuries. ${ }^{1}$ The first formalized collaboration of physicians caring for patients with vascular anomalies was in 1976, organized by Anthony Young from London and John Mulliken from Boston. This group convened a scientific society for the study of vascular malformations and hemangiomas, and founded the International Society of the Study of Vascular Anomalies (ISSVA) in 1992. ISSVA continues to meet every 2 years, bringing together experts from different specialties across the world dedicated to the care of patients with vascular anomalies. The first vascular malformation and hemangioma clinics developed within the practices of surgeons, dermatologists, and other proceduralists who were the primary recipients of referrals for these patients. The first robust, multidisciplinary vascular anomalies program was developed at Boston Children's Hospital based on the work of Drs. John Mulliken, Anthony Young, Julie Glowacki, and Judah Folkman. With the development of the first classification system for vascular anomalies in $1982,{ }^{2}$ the complexity and unique management requirements of these tumors became clearer. Centers began to move towards an interdisciplinary approach, reflected both in the development of new clinical programs, as well as the inclusion of more specialists within ISSVA and other professional groups. 
One of the first major vascular anomalies program to develop within a hematology-oncology division was the Hemangioma and Vascular Malformations Center established at Cincinnati Children's Hospital in 2001. The organization of this program has been instrumental in guiding development of similar programs around the nation, primarily through establishing clear roles for clinical, leadership, support and research teams. The establishment of this program brought the role of the pediatric hematologist-oncologist in the care of vascular anomalies to the national stage. Hematologists-oncologists are well poised to take on this central role within vascular anomalies programs, bringing to the team their knowledge of chemotherapeutic agents, experience managing coagulopathy, expertise in caring for patients with complex medical and surgical needs, and robust clinical research capabilities.

\section{Benefits of Multidisciplinary Care in Vascular Anomalies}

The key goals of multidisciplinary care in vascular anomalies are to improve the care of patients and to advance the science of the field. Additional benefits include better access to specialized care, enhanced education and experience for providers, and improved revenue streams for medical centers. Vascular anomalies clinics have a particularly strong interest in providing multidisciplinary care to their patients-whose conditions tend to be uncommon and likely to benefit from the expertise of multiple subspecialists-but much of the rationale for multidisciplinary care follows from experience and research across healthcare systems. Over the last several decades multidisciplinary care has become increasingly common as a way to harness expertise from multiple subspecialists and provide more streamlined and patient-centered care. ${ }^{3,4}$ The key principles underlying development of a successful multidisciplinary team are shared goals, mutual trust, effective communication, and measurable processes and outcomes. ${ }^{5,6}$ Important considerations include developing a business plan, obtaining hospital support, creating a clear schedule and logistics plan, outlining key role/job descriptions, and developing methods for data collection and outcome tracking. ${ }^{3}$ These processes will vary from center to center depending on the resources available, but the key features should be consistent. Multidisciplinary team improvement and checklist tools are available, much adapted from oncology care, to organize and evaluate such collaborations. ${ }^{7}$

\section{Origins of Multidisciplinary Care}

The concept of multidisciplinary care for complex or rare diseases is not new, nor specific to the field of vascular anomalies. The origin of interdisciplinary healthcare teams is generally traced back to military models of healthcare developed during World War II. ${ }^{8}$ Successful implementation of multidisciplinary teams during wartime led to subsequent multidisciplinary team development in the fields of surgery, rehabilitation, burn injury, and mental health care. Development of more modern interdisciplinary healthcare teams in the US came out of various social movements and helped establish multidisciplinary care as beneficial for both patients and health professionals. ${ }^{8-11}$ A major impetus to the development of modern multidisciplinary teams came from the development of new healthcare delivery models such as healthcare maintenance organizations (HMOs) in the 1990s, encouraged also by increased fiscal pressures on hospital systems. ${ }^{8}$ In more recent years, there has been increased support for development of multidisciplinary care teams, based on recommendations from the Institute of Medicine and legislation from the Patient Protection and Affordable Care Act of 2010. ${ }^{5}$

\section{Current Models of Multidisciplinary Care}

Two primary models of the modern multidisciplinary clinic (MDC) exist, the virtual or sequential model and the concurrent model. ${ }^{12}$ Depending on existing clinic structures and resources, there are different benefits to each. The virtual or sequential model (Figure

1) includes a multidisciplinary team discussion about a patient's case, but patient visits occur separately. The coordinator for the team gathers medical information (frequently with clinical back-up from a nurse or physician) and then schedules patients to see the appropriate providers following the group discussion. In this model, patients are scheduled to see physicians with varying expertise sequentially, with each physician rendering his or her opinion as part of a final treatment plan. Physicians do not see the patient together, but rather communicate with each other between visits and then discuss a comprehensive treatment plan at a follow-up multidisciplinary conference. Benefits to this approach include taking advantage of physician's 
existing clinic space and schedules. However, this model can be cumbersome to patients and can lead to disjointed communication.

The concurrent model of multidisciplinary care (Figure 2) treats the clinic as a separate entity from each physician's primary clinics. Patients see multiple providers within a single day, either together in one room, or sequentially but within the same clinic space and time. The coordinator remains key for gathering the necessary medical information prior to clinic and ensuring appropriate scheduling. In the concurrent model, the team generally reviews patient history, radiologic scans, clinical photos, and pathology at a conference prior to or during the clinic day. Following the in-person visit(s), a treatment plan is developed and then communicated back to the patient and referring physician. Benefits to this model include improved communication and coordination of care, particularly for complex patients with multiple needs. Patients benefit from the ability to see all necessary providers on the same day and often are able to see other supportive providers (e.g. PT/OT, social work, and case managers) who are familiar with vascular anomalies. When providers with different knowledge bases and experiences work together simultaneously, there is also invaluable interprofessional learning and experience sharing. This model is frequently employed in other clinical settings requiring interdisciplinary care, such as hemophilia, bone marrow failure, and neuro-oncology clinics. ${ }^{13} \mathrm{Al}-$ though an integrated team approach can be achieved with separate office visits and frequent communication, management is more efficient and effective with an integrated approach. ${ }^{14,15}$ Patients frequently report improved satisfaction and outcomes with the integrated approach, siting the benefit of direct communication with group formation of treatment decisions at a single point in time. ${ }^{16-18}$ This integrated practice model produces sharing of knowledge and clinical expertise between the different subspecialties, creates a support staff familiar with all aspects of care, and reduces time and financial burden on the patients.

Choosing a model for a multidisciplinary clinic must take into account the needs and resources of the medical community in which is it housed. Benefits to the concurrent model include efficiency for both patients and providers, improved communication between specialists, and knowledge building as a team. However, the virtual or sequential model provides the ability for a multidisciplinary team to function effectively even in the setting of time or space constraints. Regardless of clinic model (sequential vs. concurrent), a multidisciplinary, case-based conference is essential for improving communication and education amongst the team members. Upcoming and prior cases are reviewed with their associated imaging and pathology. This conference typically includes a mixture of case management and educational components. The frequency of team meetings can be adjusted based on the caseload at each center and the availability of team members. A smaller community-based center may be adequately served with quarterly conferences, whereas a busy tertiary center may have weekly vascular anomalies team meetings. Much of the management of vascular anomalies is chronic and semi-elective, but urgent cases will arise, so the team members need to have a system in place for quick and effective communication.

\section{Assessing Needs and Resources}

The first step in planning for a vascular anomalies multidisciplinary clinic (VA-MDC) is a survey of the needs and resources of the local patient and medical community. Multidisciplinary care can be highly varied and dependent on institutional resources ${ }^{13,19,20}$ but there are some key resources that each center should strive to employ. Some are key to successful initiation of a clinic and others that can be added as the clinic and program grow (Table 1). Each group should take into account the current system of care for patients with vascular anomalies within their community and any established referral patterns. Involving key medical providers in the community and specialists helps to smooth the transition to an interdisciplinary team. If resources such as equipment, funding, or administration support currently exist, plans can be made to leverage them for the program or to share burdens where they need to be.

At each of our centers, a critical first step in developing our VA-MDCs was to meet with our institutional leaders to discuss the benefits and financial implications of establishing a VA-MDC. This was crucial to developing a sustainable program model and garnering leadership "buy-in". In each of our regions, there were not existing comprehensive vascular anomalies programs. Patients were cared for in a heterogeneous manner, based on the specialty to which they were referred. With the help of our administrative partners, 
we researched the electronic medical record to quantify the number of children cared for in the system with vascular anomalies, the specialties to which they were referred, and the mechanisms by which they were commonly treated. This analysis included imaging, pathology, dermatological, surgical and/or interventional radiology interventions, and medical management. This investigation into the mechanisms by which patients with vascular anomalies were cared for in our system revealed widely fragmented care, care plans that were heavily influenced by which service was initially consulted, and substantial lost revenue from patients being transferred to established vascular anomalies centers despite existing expertise in our system. This transfer of care resulted in lost revenue from hospital-based clinic visits, imaging, minimally-invasive procedures, and surgeries. Ultimately, our analysis demonstrated the need for a VA-MDC in our region and outlined a roadmap for the clinic's fiscal viability, which garnered support across departments.

\section{Organization and Key Roles}

The organization of a VA-MDC may vary based on the local community's needs, resources, and model employed, but there are several key features that each group should consider. This includes a core group of clinical providers, a team coordinator, a multidisciplinary conference, and a process for streamlining referrals and treatment recommendations. Recognizing that each center will start off with different resources, we provide recommendations for roles and resources that are considered essential and those that can be added as a successful program grows (Tables 1 and 2). A recent practice survey of 25 pediatric hematologistsoncologists through the American Society of Pediatric Hematology-Oncology, showed significant practice variations amongst national vascular anomalies teams (Table 3). This demonstrates how various groups have adapted to the specific needs and resources of their medical community. We also highlight a comparison between our two programs (Figure 3). The Vanderbilt program is still in a growing and developing phase, compared to the more mature and established program at Children's Healthcare of Atlanta (CHOA). Together, these examples may provide a resource and roadmap for pediatric hematologists-oncologists looking to start or grow their vascular anomalies program.

A crucial role within any VA-MDC is that of the clinic or program coordinator. Because of the multidisciplinary nature of the team, there must be at least one team member whose role is to oversee and organize care for patients. This individual must organize providers' schedules and patient appointments in order to take this burden off of the patient and family. The coordinator also facilitates conferences, manages referrals, and ensures treatment recommendations are carried forward. The role of the coordinator must be understood to be the cornerstone of the patient's experience. This person serves as the point of contact, supporter, and often cheerleader for families who are under immense stress. If the coordinator is able to conceptualize themselves as a patient advocate, they will take ownership of this patient population, reaching out to support individual patients, searching for areas of opportunity for team research and networking, and promoting the team within the healthcare system. Because care coordination is so crucial, larger programs may need more than one individual to fill this role. The background expertise for a coordinator may be variable and based upon the needs of a specific team within the framework of its institution. An individual with an administrative background can be excellent in this role but will be limited in terms of clinical knowledge and ability to appropriately triage referrals. A coordinator with a nursing background may have the added faculties to evaluate consults, place orders for imaging or lab work, and monitor clinical response to therapy. An advanced practice provider (APP), such as a nurse practitioner (NP) or physician's assistant (PA), will be able to provide more advanced support in these areas as well as the ability to conduct clinic appointments for appropriate follow up patients. These various responsibilities of the coordinator role may be filled in a titrated fashion based on the needs and resources of the institution. A center with a relatively small patient volume may require only one administrator working closely with the physician providers, whilst a high-volume center may have an administrator and clinical providers working together in coordinator roles.

A successful VA-MDC requires the involvement of a few key physician subspecialists, though this may vary depending on the relative strengths, clinical interests, and training backgrounds of involved providers. Even within smaller settings, it is essential to develop a core group of physicians to review each case, see patients with urgent issues, and refer to other specialists as the need arises. This core of providers may be distilled 
to 1) a medical provider, 2) a surgeon, and 3) an interventional radiologist. The medical provider must have experience using the various pharmaceutical treatments available to patients, knowledge of the potential medical complications of these disorders, and the ability to facilitate genetic testing and interpretation. The surgeon must be able to provide an expertise in the anatomic ramifications of vascular anomalies throughout the body and enumerate the relative risks and benefits for surgical intervention. If this individual does not perform the recommended procedures, they may have relationships with specialists in other disciplines with those pertinent skills. The interventional radiologist must be familiar with and be able to perform minimally-invasive, percutaneous, and endovascular therapies, and discuss potential risks and benefits of such procedures. If an additional diagnostic radiologist is not a member of the team, the interventional radiologist should be able to also recommend and interpret imaging of vascular anomalies and facilitate discussion within conferences in a manner similar to a tumor board. The core physician group must be committed to the concept of team care as well as to maintaining current knowledge of the rapidly evolving research and progress in the field. As the program grows, additional specialties can be incorporated into the group. While the array of specialists may be wide, it is certainly not necessary for every provider to see every patient. The roster of appointments should be tailored to each individual case. While not available at every center, there is increasing need for the involvement of a geneticist or genetic counselor within a VA-MDC. As the vascular anomalies community continues to identify more underlying molecular mechanisms, genetic testing is rapidly becoming standard of care to accurately diagnose, prognosticate, and outline therapeutic options for patients. We are confident that the role of geneticists will continue to increase within VA-MDCs over coming decades.

\section{Role of Patient Advocacy Groups}

Patient advocacy groups play a key role in educating and supporting patients with rare diseases, and this is certainly true within the field of vascular anomalies. Incorporation of local and national patient support and advocacy groups is a factor that each program should consider. Particularly in the field of oncology, there already exists a strong framework for incorporation of patient advocacy groups into the clinical enterprise as well as involvement with industry and clinical trial recruitment. ${ }^{21,22}$ Families will look to these groups for information and support. Often this relationship is beneficial to all, but at times, input from advocacy groups can differ from recommendations of the clinical team. Teams should be aware of the available support networks for patients. It can be helpful for programs to share a recommended list of patient support groups and even direct patients and parents to helpful local resources and people. Our programs have each identified local patients who are part of national advocacy groups. These patients and advocates are included in program discussions about growth and structure, outreach, and direct patient support.

\section{Research Structure}

While delivering optimal patient care is the foremost goal of all multidisciplinary clinics, VA-MDCs also offer an ideal framework to create and apply new knowledge that improves patient outcomes. A VA-MDC provides a structure to conduct clinical trials, quality improvement projects, and conduct longer-term observational studies. The structure of the VA-MDC can allow for research participant identification, collection of biologic samples, molecular testing to explore prognostic factors, and application of molecular targeted therapies. ${ }^{23,24}$ A VA-MDC is perfectly positioned to bridge the research laboratory knowledge with clinical and translational research. Research capabilities should be incorporated at the inception of the program. It is important to assess the research landscape of your institution and form partnerships in order to utilize the existing resources and research infrastructure. For example, the VA-MDC may be able to utilize an existing pediatric cancer center solid tumor biorepository for banking pathologic specimens. Such a biorepository can help facilitate identification of new genetic abnormalities in vascular anomalies, probe the molecular mechanisms involved in vascular morphogenesis/growth/development, and allow genotype-phenotype correlation studies and other translational studies. Having tissue available for research will allow for future research involvement that has the potential to revolutionize the field vascular anomalies. In addition, setting up a database to collect clinical information on patients seen in the VA-MDC will assist in future retrospective and prospective research projects. Platforms such as REDcap@ can by utilized to collect information re- 
garding diagnosis, imaging and lab results, medication management, procedures and surgeries performed. Other options to expand the research footprint of a VA-MDC is to consider becoming an affiliate member of a cooperative group, if available, as this enables a program to partner with a member institution and participate in all trials offered through the institution's cooperative group affiliation. ${ }^{23,24}$ Because of the rare nature of these disorders, a collaboration with clinical and basic scientists at other institutions may provide an additional opportunity to advance research in vascular anomalies. Careful attention should be paid to issues of authorship and grant support at the beginning of any research collaboration, taking into account the academic and financial constraints of each contributing party.

\section{Monitoring Outcomes and Defining Success}

A thoughtfully organized VA-MDC has the potential to lead to improvement in many areas, including patient clinical outcomes, quality of life, institutional finances, research advancement, and career development. Financial viability is key to garnering institutional leadership support. A system to track patient referrals to the clinic, the outcomes of those visits (including labs, imaging, and surgical procedures ordered), and the revenue generated by the clinic is key to supporting the growth of a program. This information is necessary to support the time and effort needed from various clinical providers. Depending on the size of the center, there are several options for tracking information to support financial viability. Centers may consider use of a unique department code, maintaining a running list of ICD-10 and CPT codes to track within the VA-MDC (or within individual provider clinics), monitoring referral numbers, and maintaining a patient list within the electronic health record. Each of our programs has utilized the assistance of department administrators to track financial information.

A process for tracking patient clinical outcomes is key to continuous quality improvement, as well as providing organization for involvement in clinical research. Incorporating outcomes and quality improvement measures is beneficial for patients in the VA-MDC as well as providing an opportunity for academic rigor. The use of a uniform electronic health record for patients seen in the VA-MDC, as well as the clinical and/or research database, will assist in accurate measurements of outcomes and quality. During the development phase of the VA-MDC, we recommend creating a form or flowsheet with discrete data fields for collection and a single progress note template to be used by all VA-MDC providers. At both of our programs, we have also incorporated quality of life tools into the patient intake and monitoring process. Each patient and parent completes the questionnaire at regular intervals. Having these measurement tools built into the EHR allows for prospective data collection and more rapid data analysis than is allowed with manual data abstraction. This research infrastructure also includes monitoring funding sources, tracking how funding is utilized, grant submissions, QOL and formal research projects, publication success, and member career development. An enormous benefit of the VA-MDC is the ability for research collaboration amongst a diverse group of healthcare professionals with varied expertise and experience. Teams can utilize this structure to develop a robust research collaboration, but should be mindful of the different academic and professional needs of each team member.

\section{Conclusions}

As the field of vascular anomalies grows and becomes more complex, the development of a vascular anomalies MDC can improve patients' access to care and successful coordination of care. Since many patients require both medical and surgical management, combining expertise in patient management can improve patient satisfaction as well as potentially lead to better patient outcomes. The different models of MDC each have their own advantages and can be adapted to fit the specific needs of a patient and medical provider community. As Telehealth becomes more readily incorporated into routine medical care, this may provide future opportunities for remote consultation and opportunities for specialists with time or space constraints to collaborate in patient care. This may also provide a pathway for improvement in transition to adult care, which is lacking for many patients with vascular anomalies. Regardless of mode of care delivery, central care coordination is crucial for providing a thoughtful, patient-centered approach to care. The multidisciplinary team also allows for the collaboration between expert professionals that extends beyond the boundaries of their individual specialties. This reduces the potential for miscommunication and eliminates the fragmentation of services 
that was once common in this underserved, under-recognized patient population. This collaboration also serves to increase the knowledge and experience of both subspecialists and community providers, improving the care for both current and future patients. Finally, partnerships with patient advocacy groups offer another method of extending clinical reach and improving patient-centered care.

\section{Conflict of Interest Statement:}

All authors have no direct and indirect conflict of interest.

\section{Acknowledgements:}

The authors would like to acknowledge the clinical coordinators at each of our sites for ensuring our programs run smoothly and our patients are well cared for. We also want to thank all of our patients, for whom we do this work and for whom we are forever grateful.

\section{References}

1. Mulliken JB, Burrows PE, Fishman SJ. Mulliken and Young's Vascular Anomalies Hemangiomas and Malformations . 2nd ed. University Press; 2013.

2. Mulliken JB, Glowacki J. Hemangiomas and vascular malformations in infants and children: a classification based on endothelial characteristics. Plast Reconstr Surg . 1982;69(3):412-422.

3. Meguid C, Ryan CE, Edil BH, et al. Establishing a framework for building multidisciplinary programs. $J$ Multidiscip Healthc . 2015;8:519-526.

4. Rosell L, Alexandersson N, Hagberg O, Nilbert M. Benefits, barriers and opinions on multidisciplinary team meetings: a survey in Swedish cancer care. BMC Health Serv Res . 2018;18(1):249.

5. University of Washington, Mitchell PH, Wynia MK, et al. Core Principles \& Values of Effective TeamBased Health Care. NAM Perspect . 2012;2(10).

6. Schepman S, Hansen J, Putter I de, Batenburg R, Bakker D de. The common characteristics and outcomes of multidisciplinary collaboration in primary health care: a systematic literature review. Int $J$ Integr Care . $2015 ; 15(2)$.

7. Soukup T, Lamb BW, Arora S, Darzi A, Sevdalis N, Green JS. Successful strategies in implementing a multidisciplinary team working in the care of patients with cancer: an overview and synthesis of the available literature. J Multidiscip Healthc . 2018;11:49-61.

8. Baldwin DC. Some historical notes on interdisciplinary and interprofessional education and practice in health care in the USA. 1996. J Interprof Care . 2007;21 Suppl 1:23-37.

9. Cherkasky M. The Montefiore Hospital home care program. Am J Public Health Nations Health . 1949;39(2):163-166.

10. Silver GA. Beyond General Practice: The Health Team. Yale J Biol Med . 1958;31(1):29-39.

11. Deisher RW. Use of the child health conference in the training of medical students. Pediatrics . 1953;11(5):538-543.

12. Munro AJ, Swartzman S. What is a virtual multidisciplinary team (vMDT)? Br $J$ Cancer . 2013;108(12):2433-2441.

13. Grosse SD, Schechter MS, Kulkarni R, Lloyd-Puryear MA, Strickland B, Trevathan E. Models of comprehensive multidisciplinary care for individuals in the United States with genetic disorders.Pediatrics . 2009;123(1):407-412.

14. Leeftink AG, Bikker IA, Vliegen IMH, Boucherie RJ. Multi-disciplinary planning in health care: a review. Health Syst . 2018;0(0):1-24. 
15. Osarogiagbon RU, Freeman RK, Krasna MJ. Implementing effective and sustainable multidisciplinary clinical thoracic oncology programs. Transl Lung Cancer Res . 2015;4(4):448-455.

16. Prabhu Das I, Baker M, Altice C, Castro KM, Brandys B, Mitchell SA. Outcomes of multidisciplinary treatment planning in US cancer care settings. Cancer . 2018;124(18):3656-3667.

17. Siaw MYL, Lee JY-C. Multidisciplinary collaborative care in the management of patients with uncontrolled diabetes: A systematic review and meta-analysis. Int J Clin Pract . 2019;73(2):e13288.

18. Joret MO, Osman K, Dean A, Cao C, van der Werf B, Bhamidipaty V. Multidisciplinary clinics reduce treatment costs and improve patient outcomes in diabetic foot disease. J Vasc Surg . 2019;70(3):806-814.

19. Page AJ, Cosgrove D, Elnahal SM, Herman JM, Pawlik TM. Organizing a multidisciplinary clinic. Chin Clin Oncol . 2014;3(4):43.

20. Horvath LE, Yordan E, Malhotra D, et al. Multidisciplinary care in the oncology setting: historical perspective and data from lung and gynecology multidisciplinary clinics. J Oncol Pract . 2010;6(6):e21-26.

21. Addario B. SC31.02 The Role of Patient Advocacy Groups. J Thorac Oncol . 2017;12(1):S147-S148.

22. Tripathy D. Multidisciplinary care for breast cancer: barriers and solutions. Breast J . 2003;9(1):60-63.

23. Baer A, Bechar N, Cohen G, Devine S. Basic steps to building a research program. J Oncol Pract . 2010;6(1):45-47.

24. Scoglio D, Fichera A. Establishing a successful clinical research program. Clin Colon Rectal Surg . 2014;27(2):65-70.

Figure Legends:

Figure 1. Virtual or Sequential Clinical Model for VA-MDC. The sequential model is best employed when there are institutional or individual barriers to providing concurrent care. In this model, cases are triaged by the MDC coordinator after collection of appropriate medical information. The case is presented at a virtual or in-person conference with all team members in attendance. Providers then see the patient individually within their own clinics, and a team consensus is made following individual provider visits.

Figure 2. Concurrent Clinical Model for VA-MDC. This model provides the most streamlined approach to multidisciplinary care, with all providers seeing a patient within the same clinic session. The multidisciplinary conference is still key to this approach, both in terms of organizing information ahead of visits, and for providing a uniform plan after the patient is seen. Depending on availability and patient specifics, all team members may not need to be present for each patient visit.

Figure 3. Comparison of Two Vascular Anomalies Programs Operations. This is an overview comparison between the two multidisciplinary vascular anomalies programs at Vanderbilt Children's Hospital and Children's Healthcare of Atlanta (CHOA). The Vanderbilt program is still in the development phases, whereas the CHOA program is more mature. Each program has defined a unique conference, meeting, and clinical structure that is in line with the needs and resources available at each institution. Both programs have outlined strategies for future growth through quality improvement, research, and patient advocacy.

\section{Hosted file}

10_27_20 Table 1 - Multidisciplinary Vascular Anomalies Program.pdf available at https:

//authorea.com/users/370842/articles/489360-how-we-established-a-multidisciplinary-

program-for-vascular-anomalies

\section{Hosted file}

10_27_20 Table 2 - Multidisciplinary Vascular Anomalies Program.pdf available at https: //authorea.com/users/370842/articles/489360-how-we-established-a-multidisciplinaryprogram-for-vascular-anomalies 


\section{Hosted file}

10_27_20 Table 3 - Multidisciplinary Vascular Anomalies Program.pdf available at https: //authorea.com/users/370842/articles/489360-how-we-established-a-multidisciplinaryprogram-for-vascular-anomalies

Figure 1. Virtual or Sequential Clinical Model for VA-MDC

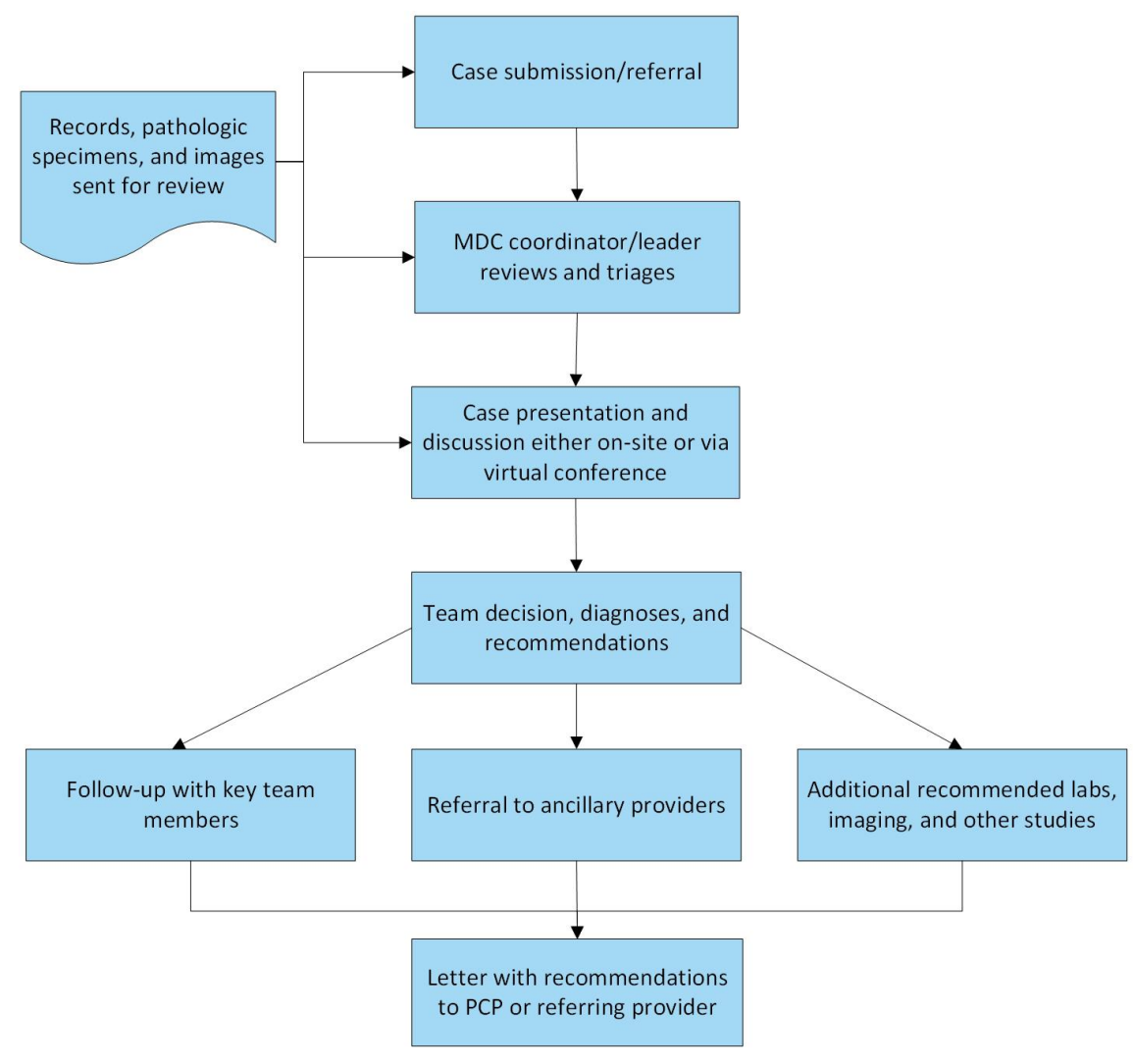


Figure 2. Concurrent Clinical Model for VA-MDC

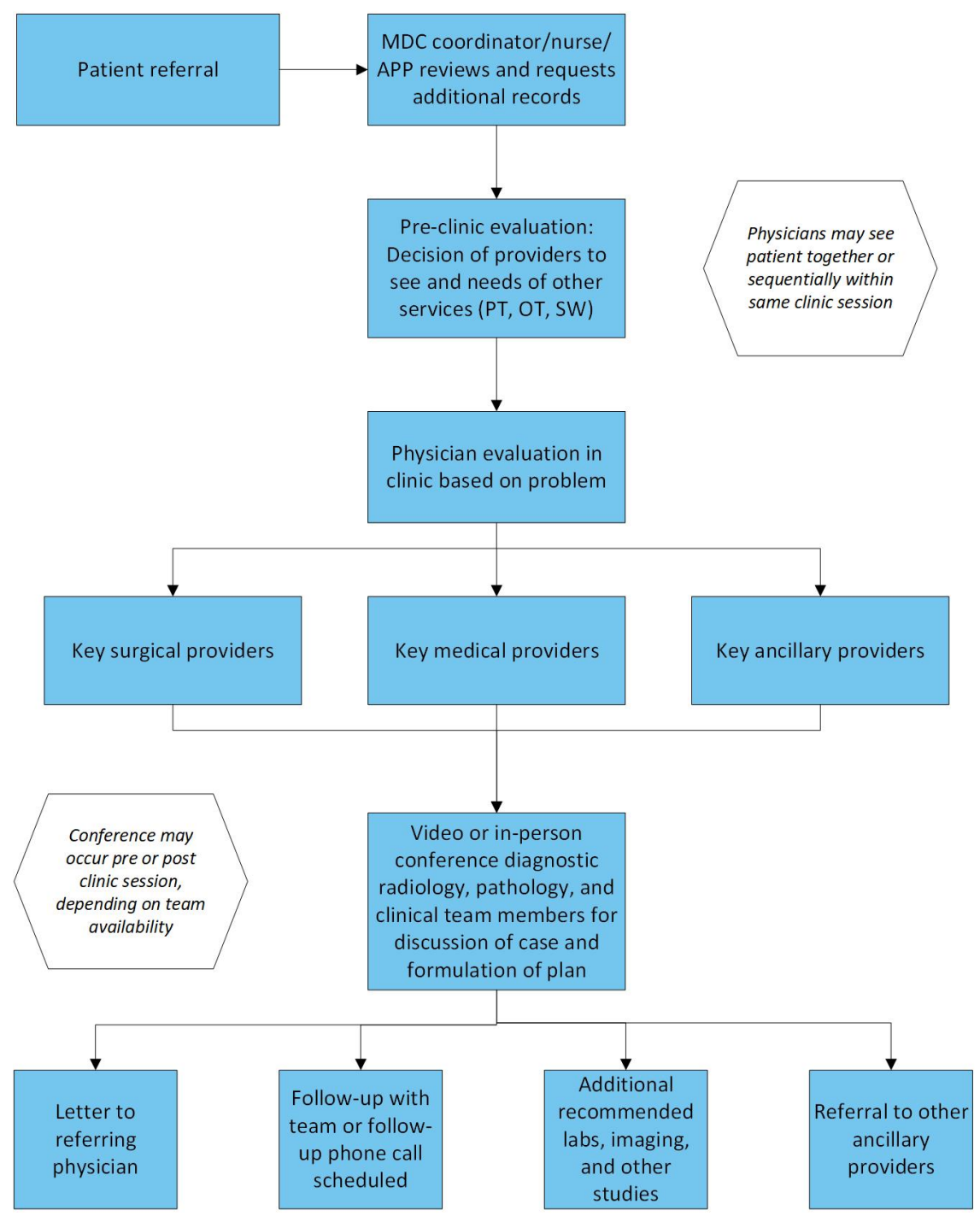


Figure 3. Comparison of Two Vascular Anomalies Programs Operations

\section{Qiiif Children's}

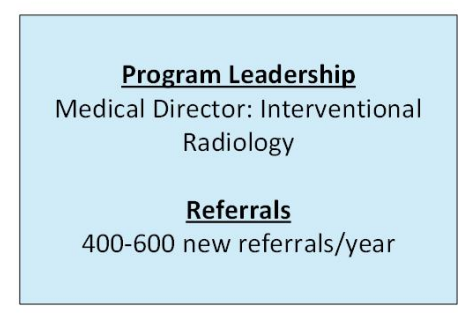

Program Meetings/Conferences

- Weekly patient review (clinicians, pathology, radiology)

- Vascular research group meeting (clinicians \& scientists) twice/year

- Program retreats and strategic meetings twice/year

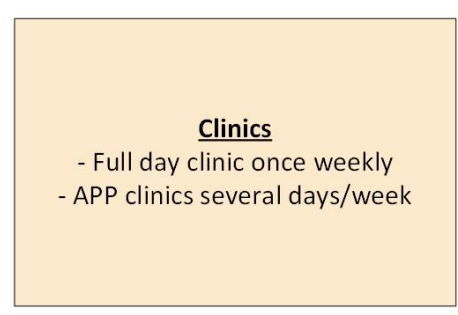

Other

- Clinical database in REDCap

- Peds Quality of Life Inventory (PedsQL)

- Tissue biorepository

- Standards of practice for all diagnoses

- Quality improvement initiatives

\section{MONROE CARELL JR. childres's Hospital a atVanderbilt}

Program Leadership

- Co-Directors: Pediatric ENT and

Pediatric Hematology-Oncology

Referrals

100 new referrals/year

Program Meetings/Conferences

- Monthly conference (clinic patients

\& outside reviews) with whole team

- Strategic meetings twice/year

\section{Clinics}

- Twice monthly clinic

- Individual providers see patients in their own clinics on additional days

\section{Other}

- Annual patient/family education day - Clinical database in REDCap - Clinical protocols for treatment of major diagnoses/medications - Incorporation into hematology clinical research team 